\title{
Effect of motorcycle helmet types on head injuries: evidence from eight level-I trauma centres in Taiwan
}

\author{
Carlos Lam ${ }^{1,2,3}$, Bayu Satria Wiratama ${ }^{3,4}$, Wen-Han Chang ${ }^{2,3,5,6,7,8}$, Ping-Ling Chen ${ }^{3}$, Wen-Ta Chiu ${ }^{3}$, Wafaa Saleh ${ }^{9}$ and \\ Chih-Wei Pai ${ }^{3^{*}}$ D
}

\begin{abstract}
Background: Motorcycle full-coverage helmet use may reduce fatalities and head injuries.

Methods: This retrospective cohort study extracted injury data from eight level-I trauma centres in Taiwan and performed a questionnaire survey to investigate injuries sustained by motorcyclists for the period between January 2015 and June 2017.

Results: As many as 725 patients participated in the questionnaire survey and reported their helmet types or phone use during crashes. The results of multivariate logistic models demonstrated that nonstandard helmet (half or open-face helmet) use was associated with an increased risk of head injuries and more severe injuries (injury severity score $\geq 8$ ). Drunk riding and phone use appeared to be two important risk factors for head injuries and increased injury severity. Anaemia was also found to be a determinant of head injuries."
\end{abstract}

Conclusions: Compared to full-coverage helmets, nonstandard provide less protection against head injuries and increased injury severity among motorcyclists.

Keywords: Motorcycle helmet type, Head injury, Motorcyclist injury severity

\section{Background}

Taiwan has an estimated population of $23,571,000$ people and an area of $36,197 \mathrm{~km}^{2}$. Here, 13,690,684 motorcycles were registered in 2017, resulting in a density of 378 motorcycles $/ \mathrm{km}^{2}$ [1]. The official statistics of Taiwan [2] demonstrated that in 2016, 51\% of road traffic fatalities involved motorcyclists. The head was the most commonly injured body region among motorcyclists in Taiwan [3, 4].

Studies [1, 5-9] have consistently concluded that helmet use is beneficial in preventing head injuries and subsequently reducing injury severity among motorcyclists. By using police-reported crash data in Taiwan, Keng [10] found that helmet use was associated with a decreased odds of deaths among motorcyclists. By linking the National Traffic Crash Data and the National Health

\footnotetext{
* Correspondence: cpai@tmu.edu.tw

${ }^{3}$ Graduate Institute of Injury Prevention and Control, College of Public Health, Taipei Medical University, 250 Wuxing Street, Taipei 11031, Taiwan Full list of author information is available at the end of the article
}

Insurance Research Database, Pai et al. [1, 3] reported that in Taiwan, helmeted motorcyclists had a decreased odds of hospitalisation due to head injuries. Furthermore, Chen and Pai [11] indicated that helmet use was beneficial in reducing head or neck injuries among motorcyclists involved in approach-turn crashes. The beneficial effects of helmet use on reducing head or neck injuries have also been well documented in other international studies $[6,7,12-14]$. Nonetheless, several studies have argued that helmet use was associated with an increased risk of neck injuries. For example, Goldstein [15] found that helmet use reduced the risk of head injuries but increased that of neck injuries in the event when speed reached more than $13 \mathrm{mph}$. Ooi et al. [16] concluded that helmet use reduced the risk of cervical spine injuries in a frontal collision, although there was an increased risk of cervical spine injuries in rear-end, side impact, and skid crashes.

Studies have attempted to examine the effects of different helmet types on head and neck injuries. For

(c) The Author(s). 2020 Open Access This article is distributed under the terms of the Creative Commons Attribution 4.0 International License (http://creativecommons.org/licenses/by/4.0/), which permits unrestricted use, distribution, and reproduction in any medium, provided you give appropriate credit to the original author(s) and the source, provide a link to the Creative Commons license, and indicate if changes were made. The Creative Commons Public Domain Dedication waiver (http://creativecommons.org/publicdomain/zero/1.0/) applies to the data made available in this article, unless otherwise stated. 
example, studies [17-22] have reported that riders wearing open-face or half helmets had an increased risk of sustaining facial and neck injuries compared with riders wearing full-face helmets. Rice et al. [23] concluded that novelty helmet or nonstandard helmet use was associated with an increased risk of fatal injuries compared with full-face helmet use after adjustment for speed and other risk factors. By using emergency room admission data in Taiwan, $\mathrm{Yu}$ et al. [24] determined that halfcoverage helmets provided poor protection from head injuries. By using Taiwan's National Head Trauma Registry data, Lam et al. [25] reported that full-coverage helmets provided the best protection from neck injuries.

The findings in most studies indicate that fullcoverage helmets are beneficial in reducing both head and neck injuries, whereas several studies have argued that helmet use was associated with an increased risk of neck injuries. To the best of our knowledge, few studies have investigated the effect of the helmet type on motorcyclist injuries in Taiwan, where motorcycle is a primary transportation mode. Some studies [4, 5, 8, 17, 24, 25] were conducted using regional hospital data, which may not provide a thorough insight into the underlying relationship between helmet types and motorcyclist injuries. In Taiwan, where motorcycles are the most common means of transportation, the number of hospitalised motorcycle-related head injuries decreased by $33 \%$ following implementation of a universal helmet law in 1997 [26]. This study investigated the effect of fullcoverage helmet use on head injuries in Taiwan using data from eight level-1 trauma centers.

\section{Methods}

\section{Study design and participants}

We conducted a retrospective cohort study in which we collected data from two sources: hospitals and individual patients. The 2-year data of inpatients (i.e. between January 2015 and June 2017) before the commencement of our research (June 2017) were retrieved from eight participating hospitals. The following eight hospitals represented at least one of the five administration districts in Taiwan: Cheng Ching Hospital Chung Kang Branch, Kuang Tien General Hospital, Mackay Memorial Hospital Taipei Branch, Mackay Memorial Hospital Tamsui Branch, National Cheng Kung University Hospital, Taipei Medical University-Shuang Ho Hospital, Taipei Medical University-Wan Fang Hospital, and Taitung Mackay Memorial Hospital. All eight hospitals are advanced level-I emergency responsibility hospitals.

To extract the data of motorcyclist patients, causes of injury for patients (in accordance with ICD-9-CM (The International Classification of Diseases, Ninth Revision, Clinical Modification) E-codes: E810.2-3, E811.2-3, E812.2-3, E813.2-3, E814.2-3, E815.2-3, E816.2-3,
E817.2-3, E818.2-3, and E819.2-3 and ICD-10-CM codes V21-V29) were used. ICD-9-CM N codes ranging from 800 to 999 that report injury diagnoses were used for extracting injury data. Disease diagnoses according to ICD-9-CM N codes or ICD-10-CM codes were used for extracting data on comorbidities. Information regarding the injury severity score (ISS) was also obtained.

The ICD-9-CM and ICD-10-CM is a modified version of World Health Organization (WHO) ICD-9 and ICD10 created by The U.S. Department of Health and Human Services to classify diseases and cause of diseases using specific codes. This study used ICD-9-CM codes from 800 to 999 and ICD-10-CM S00 - S99 codes to identify injury diagnoses in the study database. We also used ICD-9-CM E810 - E819 and ICD-10-CM V20 V29 to identify cause of injuries.

We extracted patients' ISSs [27] from the participating hospitals: ISS is calculated using the sum of squared of the highest Abbreviated Injury Score (AIS) [28] from three different injured body regions. The AIS classified anatomic body region into six parts: head, face, chest, abdomen, extremities, and external. The AIS assigns score 1 to 6 with higher score indicting a more severe injury.

After extracting patient data from the eight participating hospitals, patients were contacted through telephone, and their consent to participate in our study was obtained. A questionnaire survey was administered to obtain additional data such as the helmet type worn, and riding behaviours. Both consent forms and questionnaires were then posted to patients who agreed to participate in the study. Patients received a convenience store voucher that was worth New Taiwan Dollar (NTD) 300 (approximately US \$10) as a compensation for filling and returning the questionnaire to the research team. The following patients were not considered: those who sustained fatal injuries, those who were aged $<18$ years, those whose nationality was not Taiwanese, and those who could not read the questionnaire. By law, those aged < 18 years cannot legally ride motorcycles, and therefore were removed from our study. Foreign patients were thought to be unable to read our questionnaires in Chinese, and therefore were not included.

\section{Variables considered}

The following variables were retrieved directly from the participating hospitals: patient sex, age (four groups: < $18,18-40,41-64$, and $\geq 65$ years), drunk riding (no: blood alcohol consumption [BAC] level: $\leq 0.03 \%$ or yes: BAC level $>0.03 \%$ ), time of crash (daytime or evening/ night), previous medical history (auditory disease: deafness, Meniere's disease, or tinnitus; visual illness; myopia, presbyopia, cataract, or xerophthalmia; anaemia; or hypertension), primary injured body regions (head, chest, abdomen, or extremities), and ISS. Four age intervals (< 
$18,18-40,41-64$, and $\geq 65$ years) were initially considered but combined to two intervals $(\geq 65$ years v.s. otherwise) to increase its statistical significance in later analyses. Casualties' previous medical history (including auditory disease: deafness, Meniere's disease, or tinnitus; visual illness; myopia, presbyopia, cataract, or xerophthalmia; anaemia; or hypertension) was identified by the diagnostic codes from the participating hospitals. For example, the following codes were used for extracting anaemia: ICD-9-CM: 280; ICD-10-CM: D50. These precrash diseases were hypothesised to affect motorcyclist injury severity.

Other crucial variables not readily available from the participating hospitals were obtained from questionnaires: helmet style, crash location, protective garments used, crash type, medication before crash, and phone use. Specifications for the following variables were obtained from questionnaires: helmet use (standard: full-coverage helmet or nonstandard: open-face or half-coverage helmet), crash location (downtown or provincial highway), other protective garments (yes: protective boots or jacket were used or no: none), crash type (multivehicle or single-motorcycle crash), medication before crash (yes: had been taking medication before crash or no: none), and phone use (yes: using a phone or no: not using a phone).

\section{Analysis}

The current research focuses on head injuries sustained by motorcyclists. The distribution of head injuries and other injured body regions by a set of variables is firstly reported. Chi-squared test was performed for examining the association between independent variables and injured body regions. To minimise type II errors (failing to reject a false null hypothesis) in variable selection and biased inferences, researchers [29, 30] have suggested that the significant levels can be set much higher than conventional levels (i.e., to values of 0.20 or more). We adopted this cut-off $p$-value of 0.2 for including variables in the multivariate logistic regression model. The conventional cutoff of 0.2 has been commonly employed in the literature (e.g. [11, 31]).

ISS, another injury indicator of our interest, was classified into two levels (ISS $<8$ and ISS $\geq 8$ ). Studies (e.g., Atkinson et al. [32]) have considered ISS $\geq 8$ as an indicator of severe injuries. Similarly, the distribution of two ISS levels (ISS $<8$ and ISS $\geq 8$ ) by a set of variables is firstly reported. Choosing a specific threshold for determining severe injuries is in general arbitrary. Several studies have adopted a wide range of ISS levels for determining severe injuries, including ISS greater than 8 [33], 10 [34], or 16 [35]. We followed the ISS threshold suggested by Palmer [36] who reported that ISS levels between $>7$ and $>9$ can be considered for determining patients who require hospitalisation or intensive care in ICU.
The Chi-square test was used to examine the significant difference between the independent variables and the dependent variables. We then incorporated any variable with $p<0.2$ in the chi-square test into the multivariate analysis.

\section{Results \\ Respondent profile}

Figure 1 illustrates the sample selection process from the participating hospitals and the questionnaire survey. As shown in Fig. 1, a total of 9246 motorcyclist patients were enrolled from the eight participating hospitals. Because of time and manpower restriction, we could randomly contact 3635 of the 9246 patients. Among these 3635 patients, 623 had invalid phone numbers and 1046 did not answer calls at all. After excluding these patients ( $n=1669), 1966$ patients remained. Of these 1966 patients or their other family members successfully contacted, 6 could not read the questionnaire, 12 lived abroad, 39 were hospitalised, and 59 passed away. After excluding patients, 1385 patients agreed to participate in our questionnaire survey. Questionnaires were then posted to these 1385 patients, and as many as 870 questionnaires were posted back to our research team. We removed the following cases: patients who did not complete the questionnaire $(n=98)$, and patients who reported to be motorcycle passengers rather than riders $(n=47)$. This yielded a valid sample of 725 patients.

\section{Helmet style and head or neck injuries}

Table 1 lists the distribution of head injuries sustained by motorcyclists according to a set of variables. A vast majority of motorcyclists wore non-standard helmets (82.92\%). Approximately $9.80 \%$ of all motorcyclist casualties were elderly; $59.16 \%$ were males; and $60.38 \%$ had full-time jobs. Regarding previous medical history, 3.74, $51.25,8.34$, and $13.41 \%$ of all motorcyclist casualties were diagnosed to have auditory diseases, visual illness, anaemic, and hypertension. The majority of motorcycle crashes took place during daytime (75.96\%), in downtown areas $(94.1 \%)$, when riders were wearing protective garments $(84.16 \%)$, when riders were involved in multiple-vehicle crashes (71.99\%). Approximately 4.1\% of all motorcyclists were taking medications before crashes; $2.34 \%$ were riding under the influence of alcohol; and $3.52 \%$ were using mobile phones.

Notably, the percentage of head injuries was higher among motorcyclists wearing non-standard helmets (142; 20.17\%) than among those wearing standard helmets (18; $12.41 \%)$. Those who had anaemia had a higher risk of sustaining head injuries $(30.43 \%)$. The percentage of head injuries was higher among motorcyclists involved in dawn or night crashes $(24.47 \%)$ than among those involved in daytime crashes (16.50\%). In total, $45 \%$ of those who reported 


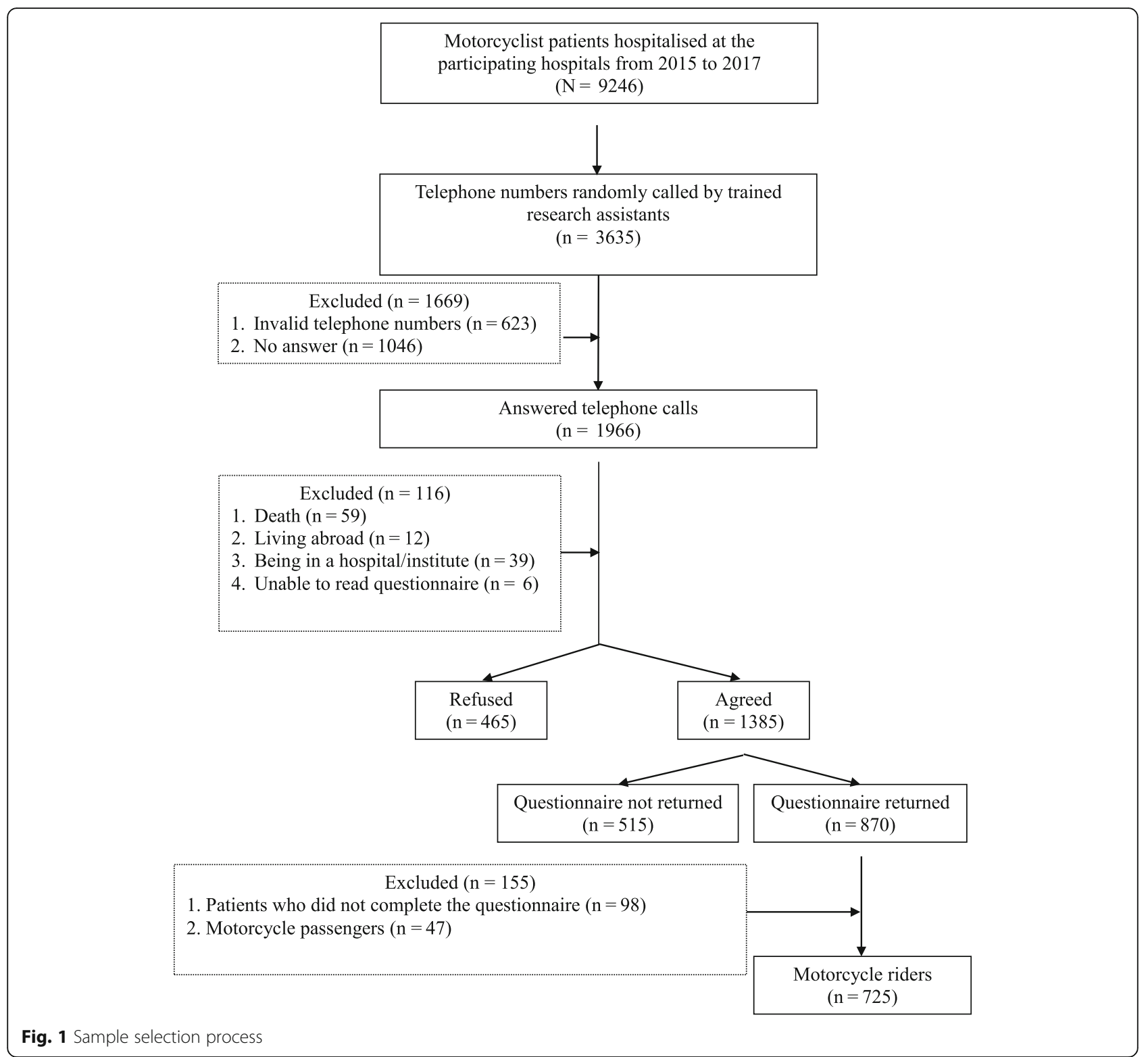

to be drinking alcohol before riding sustained head injuries, which was higher than those indicated otherwise (18.37\%). Phone use appeared to be a contributory factor to head injuries: $33.3 \%$ of those who reported to be using their phones sustained head injuries.

By using the chi-square test, we determined that the following variables were significantly associated with head injury: helmet type, motorcyclist's age, occupation, anaemia, hypertension, time of crash, alcohol consumption before riding, and mobile phone use. These variables were then incorporated into stepwise logistic regression models.

Table 2 presents estimation results obtained from stepwise logistic regression models. The estimated parameter for non-standard helmets was significant, suggesting that motorcyclists wearing non-standard helmets were 1.324 times more likely (adjusted odds ratio $[\mathrm{AOR}]=1.324$; confidence interval $[\mathrm{CI}]=1.073-1.634$ ) to sustain head injuries compared with those wearing standard helmets. Other risk factors for head injuries include having anaemia ( $\mathrm{AOR}=1.985 ; \mathrm{CI}=1.174-3.357$ ), crashes occurring at dawn or night hours $(\mathrm{AOR}=1.7$; $\mathrm{CI}=1.090-2.651)$, drinking alcohol before riding ( $\mathrm{AOR}=$ 1.823; $\mathrm{CI}=1.102-3.016$ ), and using mobile phones and riding at the same time $(\mathrm{AOR}=2.199 ; \mathrm{CI}=1.236-3.913)$.

\section{Helmet style and ISS}

Table 3 lists the distribution of severe injuries according to a set of variables. Notably, the percentage of severe injuries (ISS $\geq 8$ ) was higher among motorcyclists wearing nonstandard helmets $(306 ; 49.84 \%)$ than among those wearing 
Table 1 Distribution of injuries according to a set of independent variables

\begin{tabular}{|c|c|c|c|c|}
\hline \multirow[t]{2}{*}{ Variable } & \multirow[t]{2}{*}{ Total } & \multicolumn{2}{|l|}{ Injury location } & \multirow{2}{*}{$\begin{array}{l}P \text { - } \\
\text { value }\end{array}$} \\
\hline & & $\begin{array}{l}\text { Head/neck } \\
n(\%)\end{array}$ & $\begin{array}{l}\text { Other body part } \\
\mathrm{n}(\%)\end{array}$ & \\
\hline \multicolumn{5}{|l|}{ Helmet type } \\
\hline 1. Non-standard helmet & 704 (82.92\%) & 142 (20.17\%) & $562(79.83 \%)$ & \multirow[t]{2}{*}{0.030} \\
\hline 2. Standard helmet & $145(17.08 \%)$ & $18(12.41 \%)$ & $127(87.59 \%)$ & \\
\hline \multicolumn{5}{|l|}{ Age } \\
\hline 1. $>=65$ & $84(9.80 \%)$ & $21(25.00 \%)$ & $63(75.00 \%)$ & \multirow[t]{2}{*}{0.133} \\
\hline 2. otherwise & 773 (90.20\%) & $141(18.24 \%)$ & $632(81.76 \%)$ & \\
\hline \multicolumn{5}{|l|}{ Gender } \\
\hline 1. Male & 507 (59.16\%) & $91(17.95 \%)$ & 416 (82.05\%) & \multirow[t]{2}{*}{0.390} \\
\hline 2. Female & $350(40.84 \%)$ & $71(20.29 \%)$ & 279 (79.71\%) & \\
\hline \multicolumn{5}{|l|}{ Occupation } \\
\hline 1. Have full time job & $483(60.38 \%)$ & $84(17.39 \%)$ & $399(82.61 \%)$ & \multirow[t]{2}{*}{0.186} \\
\hline 2. Don't have full time job & 317 (39.62\%) & 67 (21.14\%) & $250(78.86 \%)$ & \\
\hline \multicolumn{5}{|l|}{ Previous medical history } \\
\hline \multicolumn{5}{|l|}{ Auditory disease } \\
\hline 1. Yes & $31(3.74 \%)$ & $7(22.58 \%)$ & $24(77.42 \%)$ & \multirow[t]{2}{*}{0.640} \\
\hline 2. No & 797 (96.26\%) & $153(19.20 \%)$ & $644(80.80 \%)$ & \\
\hline \multicolumn{5}{|l|}{ Visual illness } \\
\hline 1. Yes & $432(51.25 \%)$ & $84(19.44 \%)$ & $348(80.56 \%)$ & \multirow[t]{2}{*}{0.724} \\
\hline 2. No & 411 (48.75\%) & $76(18.49 \%)$ & $335(81.51 \%)$ & \\
\hline \multicolumn{5}{|l|}{ Anaemia } \\
\hline 1. Yes & 69 (8.34\%) & $21(30.43 \%)$ & $48(69.57 \%)$ & \multirow[t]{2}{*}{0.012} \\
\hline 2. No & 758 (91.66\%) & $137(18.07 \%)$ & $621(81.93 \%)$ & \\
\hline \multicolumn{5}{|l|}{ Hypertension } \\
\hline 1. Yes & $111(13.41 \%)$ & $27(24.32 \%)$ & $84(75.68 \%)$ & \multirow[t]{2}{*}{0.131} \\
\hline 2. No & 717 (86.59\%) & $131(18.27 \%)$ & $586(81.73 \%)$ & \\
\hline \multicolumn{5}{|l|}{ Time of traffic } \\
\hline 1. Dawn/night & 188 (24.04\%) & $46(24.47 \%)$ & $142(75.53 \%)$ & \multirow[t]{2}{*}{0.014} \\
\hline 2. Daylight & $594(75.96 \%)$ & $98(16.50 \%)$ & $496(83.50 \%)$ & \\
\hline \multicolumn{5}{|l|}{ Traffic location } \\
\hline 1. Downtown area & $654(94.10 \%)$ & $120(18.35 \%)$ & $534(81.65 \%)$ & \multirow[t]{2}{*}{0.320} \\
\hline 2. Provincial highway & 41 (5.90\%) & $5(12.20 \%)$ & $36(87.80 \%)$ & \\
\hline Other protective garments & & & & \\
\hline 1. No & $134(15.84 \%)$ & $24(17.91 \%)$ & $110(82.09 \%)$ & 0.747 \\
\hline 2. Yes & $712(84.16 \%)$ & $136(19.10 \%)$ & $576(80.90 \%)$ & \\
\hline Crash type & & & & \\
\hline 1. Multiple vehicle & 609 (71.99\%) & $114(18.72 \%)$ & $495(81.28 \%)$ & 0.737 \\
\hline 2. Single vehicle & 237 (28.01\%) & $42(17.72 \%)$ & $195(82.28 \%)$ & \\
\hline Object type & & & & \\
\hline 1. Fixed object & $30(3.52 \%)$ & $9(30.00 \%)$ & $21(70.00 \%)$ & 0.113 \\
\hline 2. Non - fixed object & $823(96.48 \%)$ & $152(18.47 \%)$ & $671(81.53 \%)$ & \\
\hline Medication before riding & & & & \\
\hline 1. Yes & 35 (4.10\%) & $9(25.71 \%)$ & $26(74.29 \%)$ & 0.301 \\
\hline
\end{tabular}


Table 1 Distribution of injuries according to a set of independent variables (Continued)

\begin{tabular}{llll}
\hline Variable & Total & $\begin{array}{l}\text { Injury location } \\
\text { Head/neck } \\
n(\%)\end{array}$ & $\begin{array}{l}\text { Other body part } \\
\text { n(\%) }\end{array}$ \\
\hline 2. No & & $153(18.70 \%)$ & $665(81.30 \%)$ \\
Drunk riding & $818(95.90 \%)$ & $9(45.00 \%)$ & $11(55.00 \%)$ \\
1. Yes (BAC level $>0.03 \%)$ & $20(2.34 \%)$ & $153(18.37 \%)$ & $680(81.63 \%)$ \\
2. No (BAC level $<=0.03 \%)$ & $833(97.66 \%)$ & & 0.006 \\
Mobile phone use & & $10(33.33 \%)$ & $20(66.67 \%)$ \\
$\begin{array}{l}\text { 1. Yes } \\
\text { 2. No }\end{array}$ & $30(3.52 \%)$ & $150(18.23 \%)$ & $673(81.77 \%)$ \\
\hline
\end{tabular}

standard helmets $(49 ; 40.16 \%)$. As many as $65.79 \%$ of elderly riders (aged 65 years or above) exhibited more severe injuries, which was statistically higher than those among their younger counterparts. Those who had anaemia had a higher risk of sustaining more severe injuries (59.32\%). The percentage of more severe injuries was higher among motorcyclists involved in multivehicle crashes (50.75\%) than among those involved in single-vehicle crashes (40.10\%). As many as $68.18 \%$ of those who reported to be drinking alcohol before riding sustained more severe injuries, which was higher than those indicated otherwise (47.49\%). Phone use appeared to be a contributory factor to more severe injuries: $65.85 \%$ of those who reported to be using their phones sustained more severe injuries.

By using the chi-squared test, we found that the following variables were significantly associated with severe injury: helmet type, motorcyclist's age, auditory disease, anaemia, crash type, medication before riding, alcohol consumption before riding, physical and mental status, and using mobile phone use. These variables were then incorporated into stepwise logistic regression models.

Table 4 presents the estimation results of the multivariate logistic regression model relating to severe injuries. The estimated parameter for non-standard helmets was significant, suggesting that motorcyclists wearing nonstandard helmet use were 1.3 times more likely $(\mathrm{AOR}=1.300$; $\mathrm{CI}=1.036-1.631)$ to sustain more severe injuries compared with those wearing standard helmets. Other risk factors for more severe injuries included elderly motorcyclists $(\mathrm{AOR}=2.116 ; \mathrm{CI}=1.213-3.690)$, those who got involved in multivehicle crashes $(\mathrm{AOR}=1.549 ; \mathrm{CI}=1.093$ 2.196), alcohol drinking before riding $(\mathrm{AOR}=1.434 ; \mathrm{CI}=$ 1.094-1.879), and using a mobile phone and riding at the same time $(\mathrm{AOR}=2.649 ; \mathrm{CI}=1.405-5.167)$.

Table $\mathbf{2}$ Odds of head or neck injuries sustained by motorcyclists

\begin{tabular}{|c|c|c|c|c|c|}
\hline Variable & $\beta$ & Standard Error & Odds Ratio & $95 \% \mathrm{Cl}$ & $P$ value \\
\hline \multicolumn{6}{|l|}{ Helmet type } \\
\hline 1. Non-standard helmet & 0.2814 & 0.1264 & 1.324 & $1.073-1.634$ & 0.0262 \\
\hline 2. Standard helmet & 1 & & & & \\
\hline \multicolumn{6}{|l|}{ Anaemia } \\
\hline 1. Yes & 0.6858 & 0.2681 & 1.985 & $1.174-3.357$ & 0.0107 \\
\hline 2. No & 1 & & & & \\
\hline \multicolumn{6}{|l|}{ Time of traffic } \\
\hline 1. Dawn/night & 0.5304 & 0.2268 & 1.700 & $1.090-2.651$ & 0.0194 \\
\hline 2. Daylight & 1 & & & & \\
\hline \multicolumn{6}{|l|}{ Drunk riding } \\
\hline 1. Yes (BAC level >0.03\%) & 0.6005 & 0.2568 & 1.823 & $1.102-3.016$ & 0.0196 \\
\hline 2. No (BAC level $\leq 0.03 \%)$ & 1 & & & & \\
\hline \multicolumn{6}{|l|}{ Using mobile phone } \\
\hline 1. Yes & 0.7880 & 0.2940 & 2.199 & $1.236-3.913$ & 0.0075 \\
\hline 2. No & 1 & & & & \\
\hline
\end{tabular}

AIC 637.394

-2 log likelihood $=625.394$ 
Table 3 Distribution of severe injuries (ISS $\geq 8$ ) according to a set of independent variables

\begin{tabular}{|c|c|c|c|}
\hline \multirow[t]{2}{*}{ Variable } & \multicolumn{2}{|c|}{ Injury Severity Score } & \multirow{2}{*}{$\begin{array}{l}P \\
\text { value }\end{array}$} \\
\hline & $\begin{array}{l}I S S \geq 8 \\
n(\%)\end{array}$ & $\begin{array}{l}\text { ISS }<8 \\
\text { n (\%) }\end{array}$ & \\
\hline \multicolumn{4}{|l|}{ Helmet type } \\
\hline 1. Nonstandard helmet & $306(49.84 \%)$ & $308(50.16 \%)$ & \multirow[t]{2}{*}{0.051} \\
\hline 2. Standard helmet & $49(40.16 \%)$ & $73(59.84 \%)$ & \\
\hline \multicolumn{4}{|l|}{ Age } \\
\hline 1. $\geq 65$ years & $50(65.79 \%)$ & $26(34.21 \%)$ & \multirow[t]{2}{*}{0.001} \\
\hline 2. Other age group & $309(46.26 \%)$ & $359(53.74 \%)$ & \\
\hline \multicolumn{4}{|l|}{ Sex } \\
\hline 1. Male & $221(49.66 \%)$ & $224(50.34 \%)$ & \multirow[t]{2}{*}{0.348} \\
\hline 2. Female & $138(46.15 \%)$ & $161(53.85 \%)$ & \\
\hline \multicolumn{4}{|l|}{ Occupation } \\
\hline 1. Full-time job & $196(46.67 \%)$ & 224 (53.33\%) & \multirow[t]{2}{*}{0.518} \\
\hline 2. No full-time job & $136(49.45 \%)$ & $139(50.55 \%)$ & \\
\hline \multicolumn{4}{|l|}{ Auditory disease } \\
\hline 1. Yes & $12(52.17 \%)$ & $11(47.83 \%)$ & \multirow[t]{2}{*}{0.152} \\
\hline 2. No & $333(48.05 \%)$ & $360(51.95 \%)$ & \\
\hline \multicolumn{4}{|l|}{ Visual illness } \\
\hline 1. Yes & 189 (50.13\%) & $188(49.87 \%)$ & \multirow[t]{2}{*}{0.839} \\
\hline 2. No & $165(46.74 \%)$ & $188(53.26 \%)$ & \\
\hline \multicolumn{4}{|l|}{ Anaemia } \\
\hline 1. Yes & $35(59.32 \%)$ & $24(40.68 \%)$ & \multirow[t]{2}{*}{0.076} \\
\hline 2. No & $311(47.26 \%)$ & $347(52.74 \%)$ & \\
\hline \multicolumn{4}{|l|}{ Hypertension } \\
\hline 1. Yes & $46(47.92 \%)$ & $50(52.08 \%)$ & \multirow[t]{2}{*}{0.954} \\
\hline 2. No & $300(48.23 \%)$ & $322(51.77 \%)$ & \\
\hline \multicolumn{4}{|l|}{ Time of traffic } \\
\hline 1. Dawn/night & $83(53.21 \%)$ & $73(46.79 \%)$ & \multirow[t]{2}{*}{0.281} \\
\hline 2. Daylight & $254(48.29 \%)$ & $272(51.71 \%)$ & \\
\hline \multicolumn{4}{|l|}{ Traffic location } \\
\hline 1. Downtown area & $269(47.28 \%)$ & $300(52.72 \%)$ & \multirow[t]{2}{*}{0.522} \\
\hline 2. Provincial highway & $20(52.63 \%)$ & $18(47.37 \%)$ & \\
\hline \multicolumn{4}{|l|}{ Other protective gears } \\
\hline 1. No & $57(49.57 \%)$ & $58(50.43 \%)$ & \multirow[t]{2}{*}{0.767} \\
\hline 2. Yes & $297(48.06 \%)$ & $321(51.94 \%)$ & \\
\hline Crash type & & & \\
\hline 1. Multiple vehicle & $272(50.75 \%)$ & $264(49.25 \%)$ & 0.011 \\
\hline 2. Single vehicle & $79(40.10 \%)$ & 118 (59.90\%) & \\
\hline Medication before riding & & & \\
\hline 1. Yes & $19(63.33 \%)$ & $11(36.67 \%)$ & 0.091 \\
\hline 2. No & $338(47.61 \%)$ & $372(52.39 \%)$ & \\
\hline Drunk riding & & & \\
\hline 1. Yes (BAC level > 0.03\%) & $15(68.18 \%)$ & $7(31.82 \%)$ & 0.056 \\
\hline 2. No (BAC level $\leq 0.03 \%)$ & 340 (47.49\%) & $376(52.51 \%)$ & \\
\hline
\end{tabular}


Table 3 Distribution of severe injuries (ISS $\geq 8$ ) according to a set of independent variables (Continued)

\begin{tabular}{llll}
\hline Variable & \begin{tabular}{l} 
Injury Severity Score \\
\cline { 2 - 3 }
\end{tabular} & $\begin{array}{l}\text { ISS } \geq 8 \\
\mathrm{n}(\%)\end{array}$ & $\begin{array}{l}\text { ISS }<8 \\
\mathrm{n}(\%)\end{array}$ \\
\hline Using a mobile phone & & $14(34.15 \%)$ \\
1. Yes & $27(65.85 \%)$ & $369(52.79 \%)$ \\
2. No & $330(47.21 \%)$ & 0.020 \\
\hline
\end{tabular}

\section{Discussion}

Studies conducted in Western countries and Taiwan [17, 20-25] have reported that full-coverage helmets protect from head injuries. In line with previous results, our results indicated that standard helmets have a highly protective role. The coverage of entire head and the presence of a chin bar explain why standard helmets provide better protection than nonstandard helmets against head injuries [23]. Our data also revealed that nonstandard helmet use was associated with an increased risk of severe injuries (ISS $\geq 8$ ). The protective effect of full-coverage helmets on head injuries reduced the overall severity of anatomic injuries. In our study, as many as $704(82.92 \%)$ motorcyclist patients wore nonstandard helmets. Our finding here underscores the importance of wearing standard helmets for reducing head injuries, particularly in Taiwan where nonstandard helmets are commonly used (e.g. 704 patients $[82.92 \%$ of our sample] wore nonstandard helmets).

Our result that nonstandard helmet use was associated with head injuries can be reasonable. This is primarily because first, nonstandard helmets provide lower protection to the heads and chins compared with standard helmets; and if the chin strap is not fully fastened, nonstandard helmets are more likely to be knocked off from a rider's head compared with standard helmets [37]. Yu et al. [24] reported that motorcyclists wearing loosely fastened helmets had a higher risk of head and brain injuries than did those wearing firmly fastened helmets. In the current research, data on whether helmets were firmly worn were not collected. Further research may attempt to investigate the relationship among injuries, helmet style, and improper helmet use.

Other findings deserve additional discussions here. For example, previous studies have examined the prevalence of mobile phone use in Mexico [38] and Vietnam [39]. Our study contributes to the motorcycle safety literature by concluding that phone use was a determinant of increased injury severity and injuries to the head. Enforcement on prohibiting motorcyclists from using their phones should be tightened.

Regarding drunk riding, studies $[1,11]$ have reported that riding under the influence of alcohol was associated with hospitalisation due to head injuries and deaths among motorcyclists. Furthermore, intoxicated riders were less likely to wear a helmet and more likely to be

Table 4 Odds of severe injuries (ISS $\geq 8$ ) sustained by motorcyclists

\begin{tabular}{|c|c|c|c|c|c|}
\hline Variable & $\beta$ & Standard Error & Odds Ratio & $95 \% \mathrm{Cl}$ & $P$ value \\
\hline \multicolumn{6}{|l|}{ Helmet type } \\
\hline 1. Nonstandard helmet & 0.2625 & 0.1157 & 1.300 & $1.036-1.631$ & 0.0241 \\
\hline 2. Standard helmet & 1 & & & & \\
\hline \multicolumn{6}{|l|}{ Age } \\
\hline 1. $\geq 65$ & 0.7496 & 0.2838 & 2.116 & $1.213-3.690$ & 0.0083 \\
\hline 2. Other age group & 1 & & & & \\
\hline \multicolumn{6}{|l|}{ Crash type } \\
\hline 1. Multiple vehicle & 0.4379 & 0.1778 & 1.549 & $1.093-2.196$ & 0.0138 \\
\hline 2. Single vehicle & 1 & & & & \\
\hline \multicolumn{6}{|l|}{ Drunk riding } \\
\hline 1. Yes (BAC level > 0.03\%) & 0.3606 & 0.1378 & 1.434 & $1.094-1.879$ & 0.0091 \\
\hline 2. No (BAC level $\leq 0.03 \%)$ & 1 & & & & \\
\hline \multicolumn{6}{|l|}{ Using a mobile phone } \\
\hline 3. Yes & 0.9910 & 0.3322 & 2.694 & $1.405-5.167$ & 0.0064 \\
\hline 4. No & 1 & & & & \\
\hline
\end{tabular}


speeding and have single-motorcycle crashes compared with nondrinking riders [40]. In accordance with the findings of these studies, we found that drunk riding was a risk factor for increased injury severity and injuries to the head. A better understanding of the relationship among motorcyclist injuries, BAC level, and traffic violation (such as speeding and helmet use) is a fruitful area for further research. The implication of our result here is that instead of motorcycle riders, driving under the influence (DUI) enforcement such as sobriety checkpoints primarily targets car drivers. To reduce alcohol-impaired riding, DUI enforcement should also target motorcycle riders.

Anaemia was found to be an important risk factor for head injuries among motorcyclist patients. Patients with anaemia are generally old and have certain chronic diseases, such as sarcopenia, and one symptom common to most types of anaemia is dizziness [41, 42]. It is likely that a driver fitness to ride a motorcycle is impaired by sarcopenia and dizziness, compelling motorcyclists to have a higher risk of head injuries once an accident has occurred. In Taiwan, by law, those who attempt to take driving- or riding-license tests are mandated to have a health checkup. In addition, labour health checkup is mandatory to employees annually or once per three to five years, depending on employee's age. The haemoglobin level is routinely checked in these health checkups. Regular screening of the haemoglobin level among those having anaemia can constitute a potential countermeasure.

A primary strength of the current research is the data we obtained from the eight level-I trauma centres, which can be more representative to the whole motorcyclist population than studies that have relied on data from a single hospital or emergency department. However, our study is limited by the binary indicator for the helmet style (standard versus nonstandard). Currently, in Taiwan, there exists no official datasets that contain data for such detailed helmet types among motorcyclist casualties. Further research may attempt to collect more detailed data on helmet types such as tropical helmets (half helmet) or openfaced helmets. Our study is also limited by the fact that all data on patients were obtained from level-I trauma centres, and as a result, a referral bias is inherently present. An important limitation of our study is that fatalities that occurred to patients within hospitals or to those who died before being transported to hospitals were not included in the analysis. Therefore, the effectiveness of helmet types in preventing fatalities and head injuries could not be established. Lastly, there are some missing data in our study which may introduce bias into the results. However, there is no significant difference between participants with and without missing data ( $p$-value $>0.05)$. Therefore, we assumed that the missing data were at least missing at random (MAR) and analysed the remaining data [43].

\section{Conclusions}

Nonstandard helmet use was associated with increased injury severity among motorcyclists after controlling for other risk factors. Furthermore, we concluded that phone use and drunk riding were two risk factors for increased injury severity and head injuries among motorcyclists. We recommend that motorcyclists should be encouraged to wear standard helmets instead of nonstandard helmets. Furthermore, we also recommend an increased enforcement on the use of mobile phone and DUI enforcement should target motorcycle riders and not only car drivers. The need for public awareness on the use of standard helmet, and the role of drunk riding and phone use while riding should be emphasised.

\section{Abbreviations}

AOR: Adjusted odds ratio; BAC: Blood alcohol consumption; Cl: Confidence interval; DUI: Driving under influence; ICD: International classification of disease; ISS: Injury severity score; OR: Odds ratio; US: United States

\section{Acknowledgments}

Our thanks go to all those who participated. This manuscript was edited by Wallace Academic Editing.

\section{Authors' contributions}

$\mathrm{CL}$ reviewed the literature, interpreted the data, and edited the English. BSW analysed the data. WHC reviewed the literature. WTC and WS assisted with interpreting the results. PLC assisted analysing and interpretation of data. CWP was responsible for study design, contributed to the analysing and interpretation of data, and drafted the manuscript. The final version of the manuscript was read and approved by each contributing author.

\section{Funding}

This research was jointly supported by the Institute of Transportation, Ministry of Transportation and Communications, Executive Yuan, Taiwan (Grant numbers: MOTC-IOT-104-SEB009 and MOTC-IOT-105-SEB010), Taipei Medical University-Wan Fang Hospital (Grant number:108TMU-WFH-14), Ministry of Science and Technology, Taiwan (Grant number: MOST 108-2221-E038-001), and Mitsui Sumitomo Insurance Welfare Foundation. The funders had no role in the design of the study, data collection and analysis, and interpretation of data or preparation of the manuscript.

\section{Availability of data and materials}

Because of the contract with Institute of Transportation, Ministry of Transportation and Communications, the original data cannot be made publicly available. As the ethical and legal restrictions from institutional review boards (IRB), data are available for researchers who meet the criteria for access. However, interested researchers may make additional data access requests to the IRB of participating hospitals at: tmujirb@gmail.com for Taipei Medical University; mmhirb82@gmail.com for Mackay Memorial Hospital; irb@ktgh.com.tw for Kuang Tien General Hospital; IRB@ccgh.com.tw for Cheng Ching Hospital Chung Kang Branch; and em73635@mail.hosp.ncku. edu.tw for National Cheng Kung University Hospital.

\section{Ethics approval and consent to participate}

The interviewers explained the study to participants in greater detail. Participants were then asked to sign a consent form acknowledging their understanding of the research. All participants consented to participate in the study, provided that no personal information, images, or video footage were revealed. Written consent was obtained from each participant. This study was approved by the institutional review boards of participating hospitals: N201510012 for Taipei Medical University; 16MMHIS168e for Mackay Memorial Hospital (Taipei, Tamsui, and Taitung Branch); J1602 for Kuang Tien General Hospital; HP17001 for Cheng Ching Hospital Chung Kang Branch; and A-ER-105-401 for National Cheng Kung University Hospital. 


\section{Consent for publication}

Neither personal images nor details of individuals are reported within the manuscript. Consent for publication was therefore not requested.

\section{Competing interests}

The authors declare that they have no competing interests.

\section{Author details}

${ }^{1}$ Emergency Department, Department of Emergency and Critical Care Medicine, Wan Fang Hospital, Taipei Medical University, 111 Xinglong Road, Section 3, Taipei 11696, Taiwan. ${ }^{2}$ Department of Emergency Medicine, School of Medicine, College of Medicine, Taipei Medical University, 250 Wuxing Street, Taipei 11031, Taiwan. ${ }^{3}$ Graduate Institute of Injury Prevention and Control, College of Public Health, Taipei Medical University, 250 Wuxing Street, Taipei 11031, Taiwan. ${ }^{4}$ Department of Epidemiology, Biostatistics and Population Health, Faculty of Medicine, Public Health and Nursing, Universitas Gadjah Mada, JL. Farmako, sekip utara sleman district, Yogyakarta 55281, Indonesia. ${ }^{5}$ Department of Medicine, Mackay Medical College, 46 Zhongzheng Road, Section 3, New Taipei 25245, Taiwan. ${ }^{6}$ Department of Emergency Medicine, Mackay Memorial Hospital, 92 Zhongshan North Road, Section 2, Taipei 10449, Taiwan. 'Mackay Medicine, Nursing and Management College, 92 Shengjing Road, Taipei 11260, Taiwan. ${ }^{8}$ Institute of Mechatronic Engineering, National Taipei University of Technology, 1

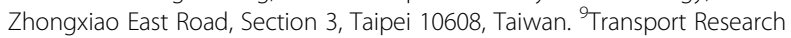
Institute, Edinburgh Napier University, Edinburgh, Scotland.

\section{Received: 24 September 2019 Accepted: 9 January 2020 Published online: 17 January 2020}

\section{References}

1. Pai CW, Lin HY, Tsai SH, Chen PL. Comparison of traffic-injury related hospitalisation between bicyclists and motorcyclists in Taiwan. PLoS One. 2018;13:1-17.

2. Ministry of Transportation and Communications. Traffic Statistics of Year 2016. Ministry of Transportation and Communications, Taiwan, Republic of China 2017.

3. Pai CW, Chen YC, Lin HY, Chen PL. A population-based case-control study of hospitalisation due to head injuries among bicyclists and motorcyclists in Taiwan. BMJ Open. 2017;7:e108574.

4. Hsieh $\mathrm{CH}$, Hsu SY, Hsieh HY, Chen YC. Differences between the sexes in motorcycle-related injuries and fatalities at a Taiwanese level I trauma center. Biom J. 2017:40:113-20.

5. Rice TM, Troszak L, Ouellet JV, Erhardt T, Smith GS, Tsai BW. Motorcycle helmet use and the risk of head, neck, and fatal injury: revisiting the hurt study. Accid Anal Prev. 2016;91:200-7.

6. Kuo SCH, Kuo PJ, Rau CS, Chen YC, Hsieh HY, Hsieh CH. The protective effect of helmet use in motorcycle and bicycle accidents: a propensity score-matched study based on a trauma registry system. BMC Public Health. 2017;17:1-10

7. Khor D, Inaba K, Aiolfi A, Delapena S, Benjamin E, Matsushima K, et al. The impact of helmet use on outcomes after a motorcycle crash. Injury. 2017;48: 1093-7.

8. Kim S, Ro YS. Shin S Do, song KJ, Hong KJ, Jeong J. preventive effects of motorcycle helmets on intracranial injury and mortality from severe road traffic injuries. Am J Emerg Med. 2018;36:173-8.

9. Liang CC, Liu HT, Rau CS, Hsu SY, Hsieh HY, Hsieh CH. Motorcycle-related hospitalization of adolescents in a level I trauma center in southern Taiwan: a cross-sectional study. BMC Pediatr. 2015;15:1-8.

10. Keng SH. Helmet use and motorcycle fatalities in Taiwan. Accid Anal Prev. 2005;37:349-55.

11. Chen PL, Pai CW. Evaluation of injuries sustained by motorcyclists in approach-turn crashes in Taiwan. Accid Anal Prev. 2019;124:33-9.

12. Erhardt T, Rice TM, Troszak L, Zhu M. Motorcycle helmet type and the risk of head injury and neck injury during motorcycle collisions in California. Accid Anal Prev. 2016;86:23-8.

13. Crompton JG, Bone C, Oyetunji T, Pollack KM, Bolorunduro O, Villegas C, et al. Motorcycle helmets associated with lower risk of cervical spine injury: debunking the myth. J Am Coll Surg. 2011;212:295-300.

14. Page PS, Wei Z, Brooks NP. Motorcycle helmets and cervical spine injuries: a 5-year experience at a level 1 trauma center. J Neurosurg Spine. 2018;28: $607-11$.
15. Goldstein JP. The effect of motorcycle helmet use on the probability of fatality and the severity of head and neck injuries: a latent variable framework. Eval Rev [Internet]. 1986;10(3):355-75.

16. Ooi SS, Wong SV, Yeap JS, Umar R. Relationship between cervical spine injury and helmet use in motorcycle road crashes. Asia-Pacific J Public Heal. 2011:23:608-19.

17. Tsai YJ, Wang JD, Huang WF. Case-control study of the effectiveness of different types of helmets for the prevention of head injuries among motorcycle riders in Taipei. Taiwan Am J Epidemiol. 1995;142:974-81.

18. Hitosugi M, Shigeta A, Takatsu A, Yokoyama T, Tokudome S. Analysis of fatal injuries to motorcyclists by helmet type. Am J Forensic Med Pathol. 2004;25:125-8.

19. Brewer BL, Diehl AH III, Johnson LS, Salomone JP, Wilson KL, Atallah HY, et al. Choice of motorcycle helmet makes a difference: a prospective observational study. J Trauma Acute Care Surg. 2013;75:88-91.

20. Ramli R, Oxley J, Hillard P, Mohd Sadullah AF, McClure R. The effect of motorcycle helmet type, components and fixation status on facial injury in Klang Valley, Malaysia: a case control study. BMC Emerg Med. 2014;14:1-11.

21. Cini MA, Prado BG, Hinnig PDF, Fukushima WY, Adami F. Influence of type of helmet on facial trauma in motorcycle accidents. Br J Oral Maxillofac Surg. 2014;52:789-92.

22. Sung KM, Noble J, Kim SC, Jeon HJ, Kim JY, Do HH, et al. The preventive effect of head injury by helmet type in motorcycle crashes: a rural Korean single-center observational study. Biomed Res Int. 2016;2016.

23. Rice TM, Troszak L, Erhardt T, Trent RB, Zhu M. Novelty helmet use and motorcycle rider fatality. Accid Anal Prev. 2017;103:123-8.

24. Yu WY, Chen CY, Chiu WT, Lin MR. Effectiveness of different types of motorcycle helmets and effects of their improper use on head injuries. Int J Epidemiol. 2011;40:794-803.

25. Lam C, Lin MR, Chu SF, Tsai SH, Bai CH, Chiu WT. The effect of various types of motorcycle helmets on cervical spine injury in head injury patients: a multicenter study in Taiwan. Biomed Res Int. 2015;2015:1-7.

26. Chiu WT, Kuo CY, Hung CC, Chen M. The effect of the Taiwan motorcycle helmet use law on head injuries. Am J Public Health. 2000;90:793.

27. Baker SP, O'Neill B, Haddon W Jr, Long WB. The injury severity score: a method for describing patients with multiple injuries and evaluating emergency care. J Trauma Acute Care Surg. 1974:14:187-96.

28. Gennarelli TA, Wodzin E, Barrington IL. Association for the Advancement of automotive medicine: the abbreviated injury scale 2005 update 2008; 2008.

29. Maldonado G, Greenland S. Simulation study of confounder-selection strategies. Am J Epidemiol. 1993;138:923-36.

30. Mickey RM, Greenland S. The impact of confounder selection criteria on effect estimation. Am J Epidemiol. 1989;129:125-37.

31. Chien DK, Hwang HF, Lin MR. Injury severity measures for predicting returnto-work after a traumatic brain injury. Accid Anal Prev. 2017;98:101-7.

32. Atkinson T, Gawarecki L, Tavakoli M. Paired vehicle occupant analysis indicates age and crash severity moderate likelihood of higher severity injury in second row seated adults in frontal crashes. Accid Anal Prev. 2016;89:88-94.

33. Hannan EL, Waller CH, Farrell LS, Rosati C. Elderly trauma inpatients in New York state: 1994-1998. J Trauma Acute Care Surg. 2004;56:1297-304.

34. Petri RW, Dyer A, Lumpkin J. The effect of prehospital transport time on the mortality from traumatic injury. Prehosp Disaster Med. 1995:10:24-9.

35. Tsai MC, Chan SH, Chang TW, Wu MH. Comparison of two different trauma assessment scores in predicting trauma outcome. J Formos Med Assoc. 1993;92:463-7.

36. Palmer C. Major trauma and the injury severity score-where should we set the bar? In: Annual Proceedings/Association for the Advancement of Automotive Medicine. Association for the Advancement of Automotive Medicine; 2007. p. 13.

37. Richter M, Otte D, Lehmann U, Chinn B, Schuller E, Doyle D, et al. Head injury mechanisms in helmet-protected motorcyclists: prospective multicenter study. J Trauma Acute Care Surg. 2001;51:949-58.

38. Pérez-Núñez R, Hidalgo-Solórzano E, Vera-López JD, Lunnen JC, Chandran A, Hijar M, et al. The prevalence of mobile phone use among motorcyclists in three Mexican cities. Traffic Inj Prev. 2014;15:148-50.

39. Truong LT, Nguyen HTT, De Gruyter C. Mobile phone use among motorcyclists and electric bike riders: a case study of Hanoi. Vietnam Accid Anal Prev. 2016;91:208-15.

40. Peek-Asa C, Kraus JF. Alcohol use, driver, and crash characteristics among injured motorcycle drivers. J Trauma Acute Care Surg. 1996;41:989-93.

41. Lam C, Kuan CF, Miser J, Hsieh KY, Fang YA, Li YC, et al. Emergency department utilization can indicate early diagnosis of digestive tract 
cancers: a population-based study in Taiwan. Comput Methods Prog Biomed. 2014;115:103-9.

42. Beghé C, Wilson A, Ershler WB. Prevalence and outcomes of anemia in geriatrics: a systematic review of the literature. Am J Med. 2004;116:3-10.

43. Kang H. (2013) the prevention and handling of the missing data. Korean J Anesthesiol. 2013;64:402-6.

\section{Publisher's Note}

Springer Nature remains neutral with regard to jurisdictional claims in published maps and institutional affiliations.

Ready to submit your research? Choose BMC and benefit from:

- fast, convenient online submission

- thorough peer review by experienced researchers in your field

- rapid publication on acceptance

- support for research data, including large and complex data types

- gold Open Access which fosters wider collaboration and increased citations

- maximum visibility for your research: over $100 \mathrm{M}$ website views per year

At $B M C$, research is always in progress.

Learn more biomedcentral.com/submissions 